\title{
Cognitive symptoms in major depressive disorder: associations with clinical and functional outcomes in a 6-month, non-interventional, prospective study in China
}

This article was published in the following Dove Press journal:

Neuropsychiatric Disease and Treatment

\begin{abstract}
Gang Wang'
Tian-Mei $\mathrm{Si}^{2}$

Lingjiang $\mathrm{Li}^{3,4}$

Yiru Fang 5

Chun-Xue Wang ${ }^{6}$

Li-Na Wang ${ }^{7}$

Kristin Hui Xian $\operatorname{Tan}^{8}$

Anders Ettrup 9

Hanne-Lise Falgreen Eriksen ${ }^{9}$

Si Luo ${ }^{10}$

Lan $\mathrm{Ge}^{10}$

'Beijing An Ding Hospital, Capital Medical University, China National Clinical Research Center for Mental Disorders, Beijing, People's Republic of China; ${ }^{2}$ Peking University Sixth Hospital \& Peking University Institute of Mental Health, Beijing, I00191, People's Republic of China; ${ }^{3}$ Key Laboratory of Mental Health, Ministry of Health (Peking University) \&National Clinical Research Center for Mental Disorders (Peking University Sixth Hospital), Beijing, I00191, People's Republic of China; ${ }^{4}$ The Second Xiangya Hospital of Central South University, Changsha, People's Republic of China; ${ }^{5}$ Shanghai Mental Health Center, Shanghai Jiao Tong University School of Medicine, Shanghai, People's Republic of China; ${ }^{6}$ Beijing Tian Tan Hospital, Department of Neuropsychiatry and Behavioral Neurology, Capital Medical University, National Clinical Research Center for Neurological Disease, Beijing, People's Republic of China; ${ }^{7}$ Tianjin Anding Hospital, Tianjin, People's Republic of China; ${ }^{8}$ Lundbeck Singapore Pte Ltd, Singapore; ${ }^{9} \mathrm{H}$. Lundbeck A/S, Valby, Denmark; ${ }^{10}$ Lundbeck China, Beijing, People's Republic of China
\end{abstract}

Correspondence: Anders Ettrup Ottiliavej 9, Valby, 2500, Denmark, Fax +45 30832204

Email aett@lundbeck.com
Objective: Cognitive symptoms in major depressive disorder (MDD) are common and may negatively impact clinical and functional outcomes. The Prospective Research Observation to Assess Cognition in Treated patients with MDD (PROACT) study aimed to assess the prevalence and course of cognitive symptoms, and their associations with clinical and functional outcomes during 6 months of antidepressant treatment, in a real-world setting among Chinese patients with MDD.

Patients and methods: Outpatients $(\mathrm{n}=598)$ aged 18-65 years with MDD and a total score $\geq 17$ on the Hamilton Depression Rating Scale - 17 Items $\left(H A M-D_{17}\right)$ were observed over 6 months after initiating new antidepressant monotherapy, with follow-up visits at months 1,2 , and 6. Cognitive symptoms were assessed using the Perceived Deficits Questionnaire Depression (PDQ-D) and cognitive performance using the Digit Symbol Substitution Test (DSST).

Results: At baseline, $76.9 \%$ of patients had indications of cognitive symptoms (PDQ-D total score $\geq 21$ ); at month 6 , this was reduced, but still present in $32.4 \%$. Across the 6 -month study period, patients improved across cognitive, clinical and functional assessments. High levels of cognitive symptoms (PDQ-D) consistently predicted worse clinical outcomes, ie, lower odds for remission and increased odds for relapse, as well as worse patient-reported functional outcomes and lower quality of life. In contrast, cognitive performance (DSST) predicted performance-based functioning but only a few patient-reported functional outcomes (absenteeism and quality of life), and no clinical outcomes. PDQ-D and DSST scores were uncorrelated at baseline.

Conclusion: The study highlights the importance of assessing and targeting cognitive symptoms for increasing patients' chances of recovery and restoring functioning in the treatment of MDD. The results further highlight the relevance of complementary assessment methods to fully capture aspects of cognitive symptoms in patients with depression.

Keywords: MDD, antidepressants, cognitive performance, real-world evidence, PDQ-D, DSST

\section{Introduction}

Major depressive disorder (MDD) is a serious and disabling disorder, and the leading cause of mental health disability worldwide. ${ }^{1}$ In China, the estimated lifetime prevalence of MDD is approximately 3.5\%. ${ }^{2,3}$ This may even likely be an underestimation, considering the substantially higher estimated lifetime prevalence of MDD reported 
internationally; ie, a meta-analysis reported an aggregate lifetime prevalence of $10.8 \%$ for 13 studies conducted worldwide from 1994 to 2013 . $^{4}$

The symptomatology of MDD is heterogeneous, comprising emotional (eg, sadness; anhedonia), physical (eg, loss of energy; sleep disturbances), and cognitive symptoms. ${ }^{5,6}$ Cognitive symptoms, typically affecting patients' processing speed, or ability to concentrate or make decisions, are listed in the diagnostic criteria for MDD in both ICD-10 ${ }^{7}$ and DSM-5. ${ }^{5}$ In a cross-sectional study conducted in Asia of more than 500 pharmacologically non-treated outpatients with depression, approximately $80 \%$ reported memory and/or concentration problems. ${ }^{8}$ Further, cognitive symptoms are common not only in the acute phase of a depressive episode but may persist during partial or even full remission for depressive symptoms. ${ }^{9}$

Still, compared to cognitive symptoms in other psychiatric disorders, the presence and impact of cognitive symptoms in MDD tend to be under-recognized in clinical practice. ${ }^{10}$ Cognitive symptoms have been shown to predict clinical outcomes in terms of course of illness and rates of response and remission, ${ }^{11,12}$ as well as patients' prognoses for restoring social and occupational functioning. ${ }^{13,14}$ For example, reduced workplace performance associated with MDD is accounted for by cognitive symptoms more strongly than by depressive symptoms. ${ }^{15,16}$ Untreated or residual cognitive symptoms in depression may, therefore, hinder complete recovery, thus increasing the negative impact on the health and daily functioning for the individual patient, as well as the health-care burden and productivity loss for society. ${ }^{17}$

The purpose of the Prospective Research Observation to Assess Cognition in Treated MDD patients (PROACT) study was to assess the prevalence and course of cognitive symptoms, and their associations with clinical and functional outcomes during 6 months of antidepressant treatment in a real-world setting among patients with MDD in China. This is the first real-world study conducted in China to characterize cognitive symptoms in MDD. Using a wide range of assessments in a large sample of patients with MDD over an extended period of time, the study allows for a detailed description of the course and temporal associations across outcome measures.

\section{Materials and methods}

\section{Patients and study design}

In this non-interventional, prospective, multi-site study, Chinese outpatients aged 18-65 years, with a diagnosis of
MDD [ICD-10] as confirmed by the Mini International Neuropsychiatric Interview and a total score $\geq 17$ on the Hamilton Depression Rating Scale - 17 Items (HAM-D ${ }_{17}$ ), were observed over 6 months after initiating new antidepressant monotherapy (first or second line), with follow-up visits at 1, 2, and 6 months in line with usual clinical practice. Exclusion criteria included any psychotic or bipolar comorbidity; alcohol or substance dependence; current prescription of more than one antidepressant or of an antipsychotic or a mood stabilizer; pregnancy; or suicidality.

All patients provided written informed consent for participation. The study was approved by the independent ethics committee of each study site. The study was conducted between March 2016 and July 2017 at a total of 15 sites in four regions of China (North, South, East, and West), in accordance with the International Conference on Harmonization Good Clinical Practices guidelines and with the ethical principles of the Declaration of Helsinki.

\section{Assessments}

Study assessments were collected at baseline and at followup visits at months 1,2, and 6. Demographic characteristics and disease history were recorded at the baseline visit. Type and eventual modification of antidepressant, other prescriptions, and manic/hypomanic symptoms were recorded at all visits. Performance-based assessments (see below) were conducted at baseline, month 2 and month 6 (ie, not at month 1) to minimize training effects.

Cognitive symptoms as perceived by the patients were assessed using the 20-item Perceived Deficits Questionnaire - Depression (PDQ-D). ${ }^{18,19}$ PDQ-D total scores are computed as the sum of scores on single items, yielding a possible score range of $0-80$, with higher scores reflecting more pronounced symptoms.

Cognitive performance was assessed using the Digit Symbol Substitution Test (DSST, not assessed at month 1), ${ }^{20,21}$ a coding task in which digits are substituted with a simple symbol. The task involves attention, processing speed, and executive functioning, and has shown sensitivity-to -change in MDD populations. ${ }^{22}$ DSST score is calculated as the total number of correct symbols within a 90 -s period (possible score range $0-133$ ), with higher scores reflecting better performance.

Depressive symptoms were evaluated by the clinicians using the HAM-D ${ }_{17},{ }^{23}$ and the Clinical Global Impressions - Severity of Illness (CGI-S), ${ }^{24}$ which records the patient's current clinical status on a scale from 1 (normal - not at all ill) to 7 (among the most extremely 
ill patients). Patients reported their depressive symptoms using the Patient Health Questionnaire-9 item (PHQ-9), ${ }^{25}$ consisting of 9 items asking for depressive symptoms during the past 2 weeks.

For HAM-D 17 [possible score range 0-52] and PHQ-9 [0-27], total scores are computed as the sum of scores on single items, with higher scores reflecting more severe depressive symptoms.

Performance-based functional capacity was assessed in a subsample of 200 patients using the University of San Diego Performance-based Skills Assessment-Brief (UPSA$\mathrm{B}$, not assessed at month 1), ${ }^{26}$ a role-play-based performance test developed for psychiatric populations, in which patients are asked to perform everyday tasks in two areas of functioning: communication (eg, calling the doctor to reschedule an appointment) and finances (eg, paying bills). Raw scores are converted to a scaled total score ranging from 0 to 100 , with higher score indicating higher functional capacity.

Functional impairment was assessed using the Sheehan Disability Scale (SDS), in which patients record the degree to which their illness interferes with functioning. From three items (work/school, social life, and family life), a total score reflecting overall functional impairment is derived [calculated only for patients with information on all three items], which ranges from 0 (unimpaired) to 30 (highly impaired).

Work productivity was assessed using the Work Productivity and Activity Impairment - Specific Health Problems Questionnaire (WPAI-SHP), ${ }^{27}$ a 6-item, patientreported scale assessing work-related impairment due to depression during the past seven days, within four dimensions: absenteeism (work time missed); presenteeism (impairment while working); overall work impairment (work productivity loss); and activity impairment. Scores for each of the dimensions are expressed as percentages, with higher scores indicating higher impairment and productivity loss.

Finally, health-related quality of life was reported by the patients using the European Quality of Life Questionnaire - 5 Dimensions (EQ-5D) ${ }^{28}$ Five items (mobility, self-care, usual activities, pain/discomfort, and depression/anxiety) are summarized in an overall utility index ranging between 0 (no problems) and 1 (extreme problems).

\section{Statistical analyses}

Analyses comprised observations for all eligible patients according to the inclusion and exclusion criteria, who had valid DSST assessments at baseline and month 2 . The prevalence of cognitive symptoms was described as the proportion of patients with PDQ-D total scores $\geq 21$ (ie, above the first quartile at baseline). The course of cognitive symptoms (PDQ-D), cognitive performance (DSST score), depressive symptoms (HAM-D 17 total score), and patient functioning (SDS total score, WPAI-SHP scores, EQ-5D utility score) were described as mean scores over time from baseline to month 6 . Because functional capacity (UPSA-B) was assessed only in a subsample $(n=200)$, this variable was not described as a main outcome.

\section{Clinical and functional outcomes}

The predictive value of PDQ-D total score and DSST score for clinical outcomes at the subsequent visit [response at month 1, remission at months 2 and 6, and relapse at month 6] was analyzed using logistic regression based on a backward model selection procedure (see Supplementary materials), with age, gender, region, HAM- $\mathrm{D}_{17}$ total score and PDQ-D total score/DSST score at the visit prior to the outcome assessment included as forced factors in all models. PDQ-D total score was categorized by the observed quartiles, and DSST score was analyzed using the following categorization: Within norm, $1 / 3$ to $2 / 3$ SD below norm, $2 / 3$ to $1 \mathrm{SD}$ below norm, or 1 SD or more below norm. ${ }^{29}$ Results from logistic regressions are presented as odds ratios versus the reference category (lowest PDQ-D quartile/DSST score within norm) with global $p$-values, with an odds ratio of 1 indicating no difference in the odds for the outcome (response/ remission/relapse).

Response was defined as a $>50 \%$ reduction (improvement) from baseline in HAM- $\mathrm{D}_{17}$ total score, while patients were considered in remission if they had a HAM- $\mathrm{D}_{17}$ total score $\leq 7$. Relapse was coded "yes" if patients had a PHQ-9 total score $\leq 9$ points at month 2 but worsened to a PHQ-9 total score $\geq 10$ points at month 6 , and "no" if PHQ-9 total score $\leq 9$ points at both month 2 and month 6 (see Supplementary materials for replacement of missing values).

The predictive value of cognitive assessments [DSST and PDQ-D at baseline and month 2] for functional outcomes [SDS total score, WPAI-SHP scores, UPSA-B total score, and EQ-5D utility index at month 2 and month 6, respectively], was analyzed using analysis of covariance (ANCOVA) with age, gender, region, HAM-D $\mathrm{D}_{17}$ total score, and PDQ-D total score/DSST score at the visit prior to the outcome assessment included as forced factors, following a backwards selection procedure (see Supplementary materials). 


\section{Relations among cognitive symptoms, depressive} symptoms, and functioning

The unadjusted associations among assessments of cognition, mood symptoms, and functioning at baseline were assessed using Pearson's correlation coefficients for each of the two cognitive assessments, PDQ-D total score and DSST score, with assessments of depressive symptoms (HAM-D ${ }_{17}$ total score) and patient functioning (SDS total score, WPAI-SHP scores, EQ-5D utility index, and UPSA-B total score).

The temporal dependencies within and across cognitive, mood, and functional domains were examined post hoc using structural equation modeling (SEM) for PDQ-D, PHQ-9, and SDS assessments across visits. A patientreported measure of mood symptoms (PHQ-9) was included in this model rather than a clinician-rated, to align assessment methodology within the model. Briefly, SEM fits a series of multivariate linear regressions modeled in a single analysis. ${ }^{30}$ In this model, the outcomes for each of the three variables were allowed to depend on the scores of all outcomes at the most recent prior visit, but not at any earlier visits, and assessments for the same visit were allowed to be correlated. Standardized regression coefficients (SRCs) are reported for the direct comparison of the strength of association across assessments and time points. Model fit was evaluated using the root-meansquare error of approximation (RMSEA), and comparative fit index (CFI) (with RMSEA $\leq 0.06$ and CFI $\geq 0.95$ considered indicative of good fit), and statistical significance of specific model parameters was evaluated using chisquared tests. All statistical tests are two-tailed and performed using SAS, Version 9.4 statistical software. SEM analyses were conducted in $\mathrm{R}$ using the "lavaan" package. ${ }^{31}$

\section{Sample size determination}

Assuming a mean DSST change from baseline of 7.6, a SD of 8.1 , and a withdrawal rate of $20 \%$, enrolling 1,000 patients would provide a relative precision of the $95 \% \mathrm{CI}$ between $93 \%$ and $107 \%$.

\section{Results}

\section{Study population}

Of 1008 patients enrolled, 666 (66.1\%) completed the 6-month visit (Table 1). A total of 598 (59.3\%) patients with valid DSST assessments at baseline and month 2 were included in the statistical analyses (analysis population). Of those analyzed, 1 patient $(0.2 \%)$ did not complete
Table I Participant flow

\begin{tabular}{|l|l|}
\hline & $\begin{array}{l}\text { Number of } \\
\text { patients }\end{array}$ \\
\hline $\begin{array}{l}\text { Total population } \\
\text { Completed the month } 6 \text { visit } \\
\text { Ineligible according to at least one inclusion/ } \\
\text { exclusion criterion }\end{array}$ & $\begin{array}{l}\mathrm{I}, 008 \\
666(66.1 \%) \\
22(2.2 \%)\end{array}$ \\
\hline $\begin{array}{l}\text { Missing visit at month 2 } \\
\text { Missing baseline DSST assessment }\end{array}$ & $385(38.2 \%)$ \\
Missing month 2 DSST assessment & $5(0.5 \%)$ \\
\hline $\begin{array}{l}\text { Analysis population } \\
\text { b,c }\end{array}$ & $16(1.6 \%)$ \\
\hline $\begin{array}{l}\text { Ldverse event to follow-up } \\
\text { Completed the 6-month observational study }\end{array}$ & $598(59.3 \%)$ \\
\hline
\end{tabular}

Notes: ${ }^{a}$ Total population: All enrolled patients who gave written informed consent. ${ }^{\text {b} A n a l y s i s ~ p o p u l a t i o n: ~ A l l ~ p a t i e n t s ~ i n ~ t h e ~ t o t a l ~ p o p u l a t i o n ~ w h o ~ w e r e ~ e l i g i b l e ~ a c c o r d-~}$ ing to study inclusion/exclusion criteria, and who had valid DSST assessments at baseline and month 2. ${ }^{\mathrm{c}} 18$ patients had more than one reason for being excluded from the analysis population. ${ }^{\mathrm{d}} \mathrm{Of}$ the analysis population.

Abbreviation: DSST, Digit Symbol Substitution Test.

the study due to an adverse event, and 50 patients $(8.4 \%)$ were lost to follow-up.

For patients included in the analyses, the mean age was 36.5 (SD $=12.0$ ) years, and nearly $70 \%$ were women (Table 2). Approximately $80 \%$ lived in urban areas and approximately $75 \%$ of the patients had an educational level of high school or higher. More than half of patients were gainfully employed or self-employed. The mean HAM- $\mathrm{D}_{17}$ total score at baseline was 23.3 ( $\mathrm{SD}=4.4$ ), indicating moderate to severe depression. Approximately $75 \%$ began first-line antidepressant treatment when entering the study, while the remainder switched from a different antidepressant. The types of antidepressants most frequently prescribed at baseline were escitalopram (32.4\%), sertraline (14.7\%), venlafaxine extended release (12.5\%), duloxetine (10.2\%), and mirtazapine (8.2\%). Baseline and clinical characteristics of the analysis population were comparable to those of the total study population.

\section{Study assessments}

The prevalence of cognitive symptoms (PDQ-D total score $\geq 21$ ) was $76.9 \%$ at baseline, $58.9 \%$ at month 1 and $44.6 \%$ at month 2 (Figure 1). At month 6, the proportion of patients with PDQ-D total score $\geq 21$ was $32.4 \%$. The mean baseline PDQ-D total score was 33.7 (SD =16.2); across study months, PDQ-D total score declined (improved) steadily to $17.7(\mathrm{SD}=15.4)$ at month $6($ Figure $2 \mathrm{~A})$. 
Table 2 Study population

\begin{tabular}{|c|c|c|c|}
\hline \multicolumn{2}{|c|}{ Sociodemographic characteristics } & \multirow{2}{*}{$\begin{array}{l}\text { Total study population }(\mathrm{N}=1008) \\
571(56.6)\end{array}$} & \multirow{2}{*}{$\begin{array}{l}\text { Analysis population }(n=\mathbf{5 9 8}) \\
357(59.7)\end{array}$} \\
\hline Region in China & North, n (\%) & & \\
\hline & South, n (\%) & $69(6.8)$ & $48(8.0)$ \\
\hline & East, n (\%) & $178(17.7)$ & $83(13.9)$ \\
\hline & West, n (\%) & $190(18.8)$ & $110(18.4)$ \\
\hline \multicolumn{2}{|c|}{ Age (years), mean $\pm S D$} & $36.0 \pm 12.0$ & $36.5 \pm 12.0$ \\
\hline \multicolumn{2}{|l|}{ Women, n (\%) } & $693(68.8)$ & $410(68.6)$ \\
\hline \multicolumn{2}{|l|}{ Married, n (\%) } & $612(60.8)$ & $371(62.0)$ \\
\hline \multicolumn{2}{|c|}{ Educational level, n (\%) } & & \\
\hline \multicolumn{2}{|c|}{ Middle school or lower } & $240(23.8)$ & $134(22.4)$ \\
\hline \multicolumn{2}{|c|}{ High school or junior college } & $321(31.8)$ & $194(32.4)$ \\
\hline \multicolumn{2}{|c|}{ University or post-graduate } & $447(44.3)$ & $270(45.2)$ \\
\hline \multicolumn{2}{|l|}{ Employed, n (\%) } & $570(56.5)$ & $343(57.4)$ \\
\hline \multicolumn{2}{|c|}{ Urban residents, $\mathrm{n}(\%)$} & $827(82.0)$ & $491(82.1)$ \\
\hline \multicolumn{2}{|l|}{ Clinical history } & & \\
\hline \multicolumn{2}{|c|}{ First-time episode, n (\%) } & $610(60.5)$ & $343(57.4)$ \\
\hline \multicolumn{2}{|c|}{ Number of previous episodes, mean \pm SD } & $2.2 \pm 2.2$ & $2.0 \pm 2.1$ \\
\hline \multicolumn{2}{|l|}{ Range, $n$} & $\mathrm{I}-20$ & $\mathrm{I}-20$ \\
\hline \multicolumn{2}{|c|}{ Duration of current episode $\geq 8$ weeks, $n(\%)$} & 725 (7I.9) & $430(71.9)$ \\
\hline \multicolumn{2}{|c|}{ Switch of antidepressant at baseline, $\mathrm{n}$ (\%) } & $219(21.7)$ & I $44(24.1)$ \\
\hline \multicolumn{4}{|c|}{ Clinical assessments, mean $\pm \mathrm{SD}$} \\
\hline \multicolumn{2}{|c|}{ PDQ-D total score } & $33.6 \pm 16.3$ & $33.7 \pm 16.2$ \\
\hline \multicolumn{2}{|l|}{ DSST score } & $50.4 \pm 16.9$ & $50.2 \pm 16.5$ \\
\hline \multicolumn{2}{|c|}{ HAM-D 17 total score } & $23.2 \pm 4.4$ & $23.3 \pm 4.4$ \\
\hline \multicolumn{2}{|l|}{ CGI-S score } & $4.6 \pm 0.8$ & $4.5 \pm 0.8$ \\
\hline \multicolumn{2}{|c|}{ SDS total score } & $17.0 \pm 7.2$ & $17.4 \pm 7.1$ \\
\hline \multicolumn{2}{|c|}{ WPAI-SHP absenteeism, \% } & $26.5 \pm 35.7$ & $26.5 \pm 34.9$ \\
\hline \multicolumn{2}{|c|}{ WPAI-SHP presenteeism, \% } & $54.8 \pm 26.8$ & $55.6 \pm 26.4$ \\
\hline \multicolumn{2}{|c|}{ EQ-5D utility index } & $0.74 \pm 0.14$ & $0.73 \pm 0.14$ \\
\hline
\end{tabular}

Abbreviations: CGI-S, Clinical Global Impressions - Severity of Illness; DSST, Digit Symbol Substitution Test; EQ-5D, European Quality of Life Questionnaire - 5 Dimensions; HAM-D 17 , Hamilton Depression Rating Scale - 17 items; PDQ-D, Perceived Deficit Questionnaire-Depression; SD, Standard deviation; SDS, Sheehan Disability Scale; WPAI-SHP, Work Productivity and Activity Impairment Questionnaire - Specific Health Problems Questionnaire.

Across the 6-month study period, the mean HAM- $\mathrm{D}_{17}$ total score decreased (improved) by $-16.4(\mathrm{SD}=6.4)$ points to a mean of $6.9(\mathrm{SD}=5.6)$ (Figure $2 \mathrm{C})$. At month $6,64.5 \%$ of patients were in remission $\left(H A M-D_{17}\right.$ total score $\leq 7)$. Similar patterns of improvement across the 6-month study period were seen across additional assessments of cognitive performance (Figure 2B) and functioning (Figure 2D-F), albeit less pronounced for cognitive performance. Overall, improvement rates were most pronounced during the first month of treatment but continued improvements were seen across the entire study period for all assessments.

\section{Baseline correlations among cognitive, mood and functional assessments}

At baseline, both cognitive symptoms (PDQ-D total score) and cognitive performance (DSST score) were significantly correlated with clinician- as well as patientreported depressive symptoms (worse cognitive scores being associated with worse mood symptoms [HAM-D 17 /PHQ-9 total scores]; Table 3); the strength of associations, however, varied substantially, particularly with patient-reported depression score (PHQ-9), for which the correlation with PDQ-D was moderate at 0.56 , while the correlation with DSST was negligible, albeit statistically 


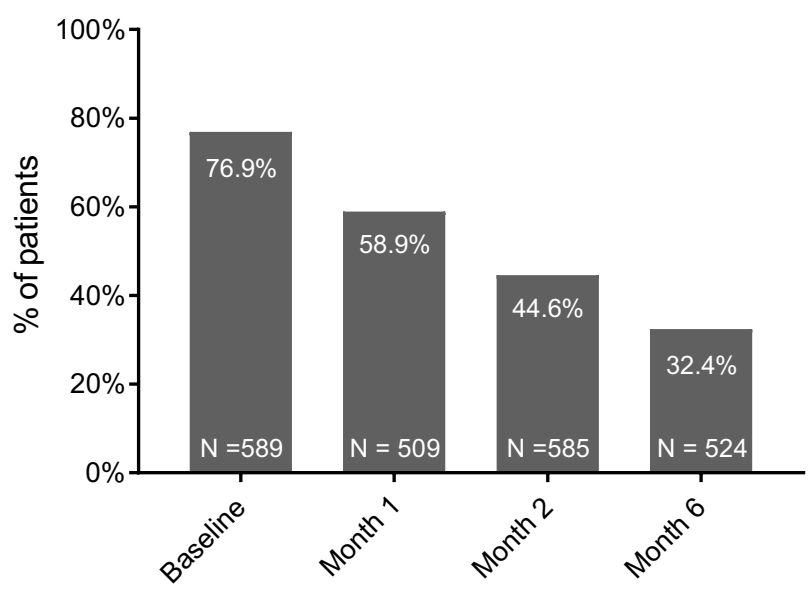

Figure I Prevalence of cognitive symptoms (PDQ-D total score $\geq 2 \mathrm{I}$ ). Abbreviations: PDQ-D, Perceived Deficits Questionnaire - Depression.

significant (Pearson's $r=-0.098,95 \%$ CI: -0.176 ; $-0.017)$.

Higher (worse) PDQ-D total score was associated with worse functioning across the range of patient-reported functional scales, except for absenteeism as measured by the WPAI-SHP, whereas higher (better) DSST score was associated with better quality of life (EQ-5D utility index) and higher functional capacity (UPSA-B total score). PDQ-D total score and DSST score were not correlated at baseline.

\section{Associations between cognitive assessments, and clinical and functional outcomes}

Higher levels of patient-reported cognitive symptoms consistently predicted worse clinical outcomes (Figure 3). Thus, patients with PDQ-D scores in the upper three quartiles at baseline had lower odds for achieving remission at month 2, compared to patients in the lowest PDQD quartile; similar associations were seen between PDQ-D score at months 2 and remission status at month 6 . Likewise, PDQ-D score predicted relapse: the higher PDQ-D quartile at month 2, the higher the odds for relapse at month 6 (Figure 3). In contrast, cognitive performance, as measured by the DSST, did not predict any of the clinical outcomes.

When controlling for mood symptoms, perceived cognitive symptoms at month 2 predicted worse outcomes at month 6 for all patient-reported functional assessments (SDS, WPAI-SHP), and quality of life (EQ-5D), but not for functional capacity (UPSA-B) (Table 4). Conversely, cognitive performance only predicted absenteeism (WPAISHP), and quality of life (EQ-5D). Further, DSST performance at baseline predicted UPSA-B performance at month 2 (result not shown); however, for month 2 DSST performance to month 6 UPSA-B performance, this association was no longer observed.

\section{Temporal interdependencies among patient-reports of cognitive symptoms, depressive symptoms, and functioning}

Assessments within each patient-reported outcome domain (cognitive symptoms [PDQ-D], depressive symptoms [PHQ-9], and overall functioning [SDS]) depended moderately to strongly on the previous assessment for the same scale, with SRC ranges of $0.63-0.75$ for PDQ-D, $0.34-0.51$ for PHQ-9, and 0.45-0.56 for SDS (all $p$-values $<0.001$; Figure 4). Consistently across timepoints, cognitive symptoms predicted depression severity as well as functional impairment, with SRCs ranging from 0.14 to 0.23 (all $p$-values $<0.05$ ). Cognitive symptoms were not predicted by depression severity nor by functional impairment at any time point.

\section{Discussion}

In this prospective real-world study, more than threefourths of acutely depressed patients had indications of cognitive symptoms (PDQ-D total score $\geq 21$ ) at the time they initiated new antidepressant treatment. At month 6, the prevalence of cognitive symptoms was reduced, but still present in nearly one-third of the patients.

During the study period, patients improved across cognitive, depressive, and functional symptom domains. Improvements in cognitive performance (DSST) appeared less pronounced compared with the overall pattern for the assessments. This finding was expected; first, none of the antidepressant treatments prescribed in the study have demonstrated level 1 evidence for improving cognitive performance $^{34,35}$ and second, cognitive performance typically does not depend on changes in mood symptoms, as indicated by the common presentation of residual cognitive symptoms among patients in remission of depressive symptoms. ${ }^{9}$ Patient-reported cognitive symptoms, on the other hand, were more strongly correlated with depressive symptoms than with cognitive performance, in line with previous findings. ${ }^{36,37}$ The absence of correlation between cognitive symptoms (PDQ-D) and cognitive performance (DSST) further substantiate that these assessment methods capture distinct aspects of cognition. ${ }^{19,38,39}$

Consistent with the differential patterns of associations seen at baseline, cognitive symptoms and cognitive performance differentially predicted later clinical and functional outcomes. Thus, PDQ-D predicted clinical outcomes 
A. PDQ-D

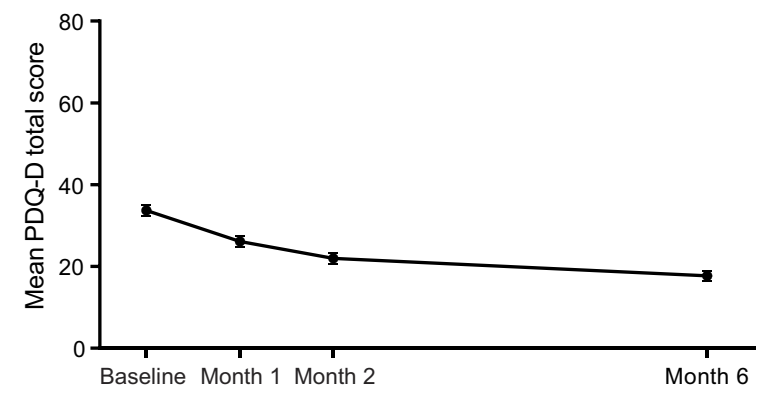

C. HAM-D $_{17}$

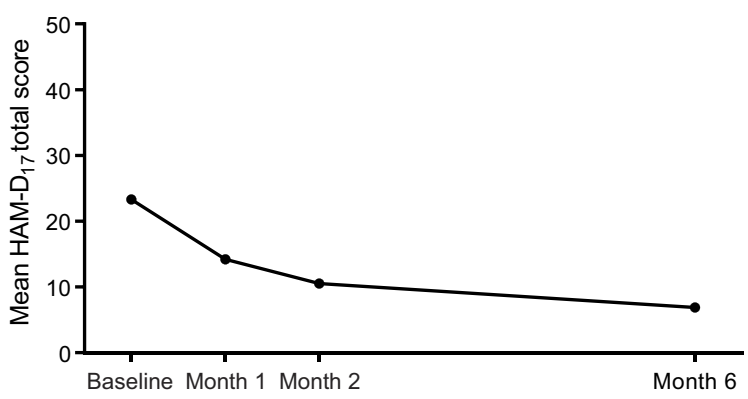

E. WPAI-SHP

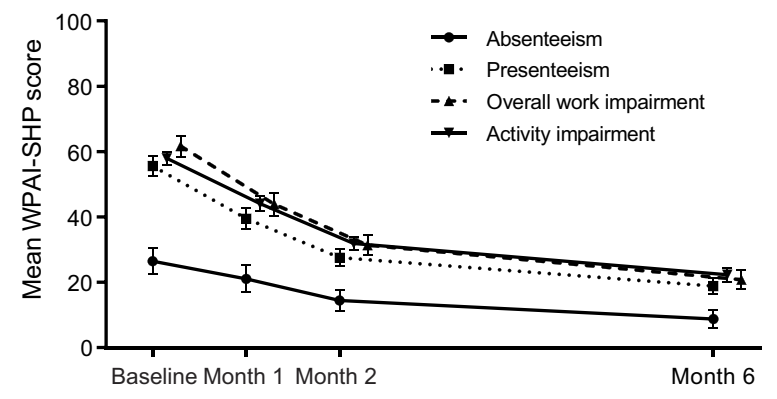

B. DSST

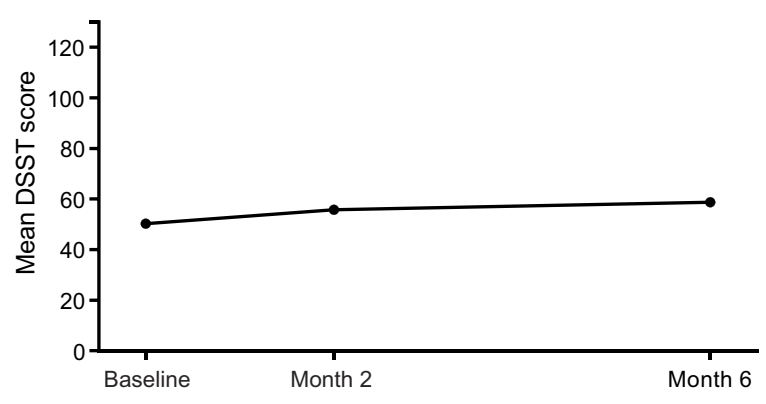

D. SDS

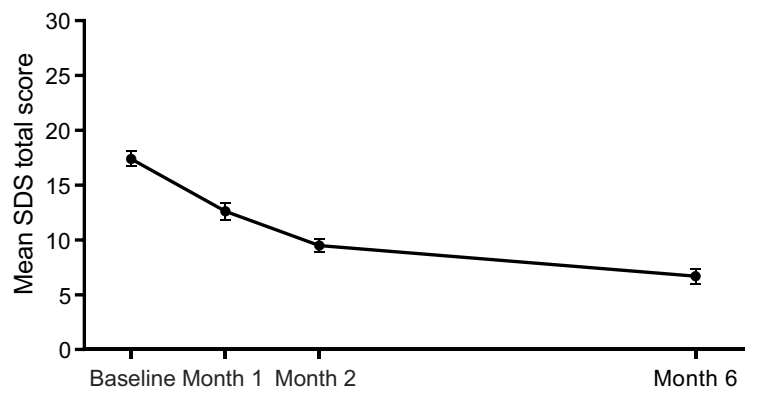

F. EQ-5D

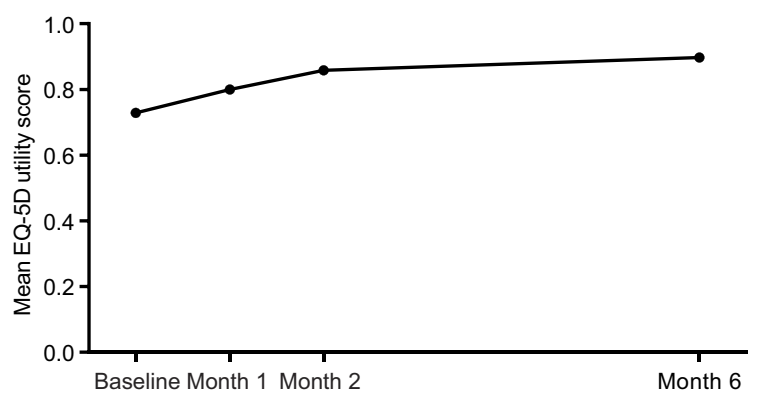

Figure 2 Study assessment across study months. Error bars represent 95\% confidence intervals. Baseline: (A) PDQ-D, $n=589 ;($ B) $D S S T, n=598 ;(C)$ HAM-D $17, n=598 ;$ (D) SDS, $n=455$; (E) WPAI-SHP absenteeism, $n=3$ I3; presenteeism, $n=278$; overall work impairment, $n=278$; activity impairment, $n=597$; (F) EQ-5D, $n=596$. Month 6: PDQ-D, $n=524$; DSST, $n=526$; HAM-D ${ }_{17},=530$; WPAI-SHP absenteeism, $n=286$; presenteeism, $n=272$; overall work impairment, $n=27 I$; activity impairment, $n=529 ;$ SDS, $n=434$; EQ-5D, $n=525$.

Abbreviations: DSST, Digit Symbol Substitution Test; EQ-5D, European Quality of Life Questionnaire - 5 Dimensions; HAM-D 17 , Hamilton Depression Rating Scale - 17 items; PDQ-D, Perceived Deficits Questionnaire-Depression; SDS, Sheehan Disability Scale; WPAI-SHP, Work Productivity and Activity Impairment - Specific Health Problems Questionnaire.

in terms of response, remission and relapse, as well as all patient-reported functional outcomes, but not performance-based functional capacity (UPSA-B). Higher baseline DSST score conversely predicted higher functional capacity at month 2 . Moreover, cognitive symptoms were more strongly associated with presenteeism, ie, work underperformance, than with absenteeism, ie, physical non-attendance. This finding is similar to the results from a European real-world study of long-term functional outcomes in depression, ${ }^{40}$ and might be explained by patients' preferences to avoid, eg, stigma or negative economic consequences associated with overt non-attendance. ${ }^{40}$ Interestingly, cognitive performance conversely predicted absenteeism but not presenteeism, underscoring the differential predictive value of perceived cognitive symptoms versus cognitive performance.

The SEM results provided further support for a causal relation among the domains of cognitive symptoms, mood symptoms, and functioning, showing that cognitive symptoms consistently predicted later patient-reported depression severity as well as functional impairment. Cognitive symptoms were not predicted by the other domains in this model. 
Table 3 Baseline correlations among assessments of cognition, mood, and functioning

\begin{tabular}{|c|c|c|c|c|}
\hline & $\mathbf{N}$ & Pearson's $r(95 \% \mathrm{CI})$ & $\mathbf{N}$ & Pearson's $r(95 \% \mathrm{Cl})$ \\
\hline & & PDQ-D total score & & DSST score \\
\hline DSST score & 589 & $-0.068[-0.148,0.013]$ & - & - \\
\hline HAM-D 17 total score & 589 & $0.212[0.134,0.288]^{*}$ & 598 & $-0.254[-0.328,-0.178]^{*}$ \\
\hline PHQ-9 total score & 588 & $0.562[0.504 ; 0.615]^{*}$ & 595 & $-0.098[-0.176 ;-0.017]^{*}$ \\
\hline SDS total score & 447 & $0.480[0.404 ; 0.548]^{*}$ & 455 & $-0.023[-0.115 ; 0.069]$ \\
\hline \multicolumn{5}{|l|}{ WPAI-SHP scales } \\
\hline Absenteeism & 308 & $0.063[-0.049 ; 0.173]$ & 313 & $-0.04 \mathrm{I}[-0.15 \mathrm{I} ; 0.070]$ \\
\hline Presenteeism & 274 & $0.430[0.327 ; 0.521]^{*}$ & 278 & $-0.056[-0.173 ; 0.062]$ \\
\hline Overall work impairment & 274 & $0.390[0.284 ; 0.486]^{*}$ & 278 & $-0.044[-0.160 ; 0.075]$ \\
\hline Activity impairment & 588 & $0.416[0.347 ; 0.48 I]^{*}$ & 597 & $-0.057[-0.136 ; 0.024]$ \\
\hline EQ-5D utility index & 587 & $-0.324[-0.395 ;-0.250]^{*}$ & 596 & $0.191[0.112 ; 0.267]^{*}$ \\
\hline UPSA-B total score ${ }^{a}$ & 103 & $-0.147[-0.331 ; 0.049]$ & 107 & $0.434[0.264 ; 0.575]^{*}$ \\
\hline
\end{tabular}

Notes: ${ }^{*} p<0.05 .{ }^{a}$ UPSA-B assessed in a subsample $(n=200)$.

Abbreviations: DSST, Symbol Digit Substitution Test; EQ-5D, European Quality of Life Questionnaire - 5 Dimensions; HAM-D 17 , Hamilton Depression Rating Scale - 17 items; PDQ-D, Perceived Deficits Questionnaire - Depression; PHQ-9, Patient Health Questionnaire-9 item; SDS, Sheehan Disability Scale; UPSA-B, University of San Diego Performance-based Skills Assessment - Brief; WPAI-SHP, Work Productivity and Activity Impairment Questionnaire - Specific Health Problems Questionnaire.

Response at month 1

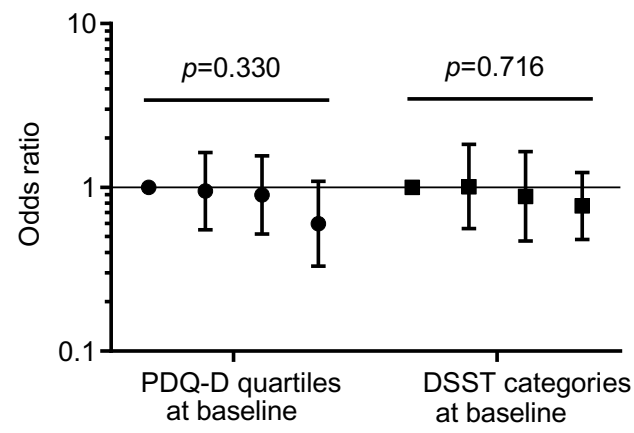

Remission at month 6

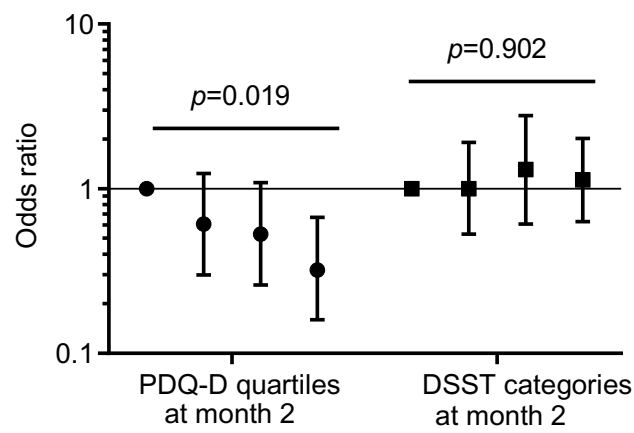

Remission at month 2

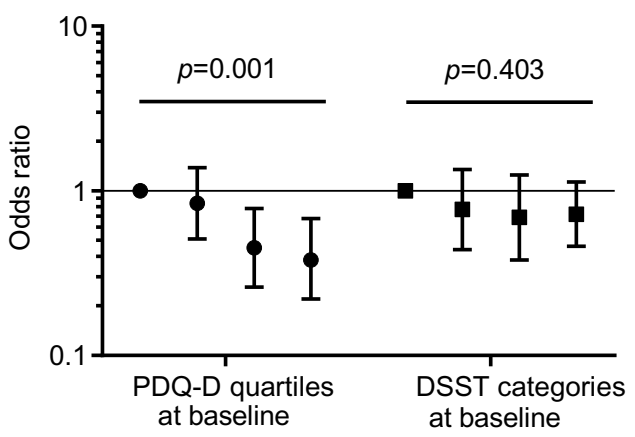

Relapse at month 6

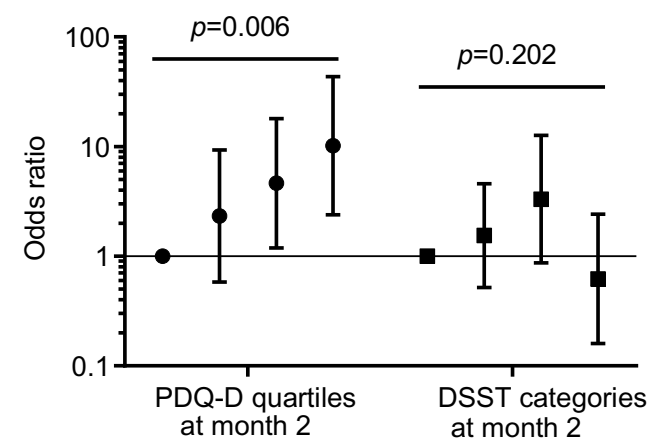

Figure 3 Associations between cognitive symptoms (PDQ-D total score)/cognitive performance (DSST score) and clinical outcomes. Reference categories (grey symbols): Lowest PDQ quartile/DSST score within norm. Logistic regression based on stepwise backward model selection with age, gender, region, HAM-D I7, and PDQ-D/DSST scores as forced factors. Note that $Y$-axis displays the log-scale (data have not been log-transformed). Error bars represent $95 \%$ confidence intervals. $P$-values based on tests for global differences. PDQ-D quartiles at baseline: $0-20, n=136 ; 21-32, n=158 ; 33-43, n=137 ; 44-80, n=158$. PDQ-D quartiles at month 2: $0-9$, $n=135 ; 10-17, n=155$; 18-30, $n=140 ; 31-80, n=155$. DSST categories at baseline: Within norm, $n=265 ; 1 / 3$ to $2 / 3$ SD below norm, $n=80 ; 2 / 3$ to I SD below norm, $n=72 ; \geq 1$ SD below norm, $n=|8|$. DSST categories at month 2: Within norm, $n=360 ; 1 / 3$ to $2 / 3$ SD below norm, $n=74 ; 2 / 3$ to I SD below norm, $n=53 ; \geq I$ SD below norm, $n=1 \mid$ I. Response defined as a reduction of $>50 \%$ from baseline in HAM-D 17 total score. Remission defined as HAM-D 17 total score $\leq 7$. Relapse: "yes" if patients had a PHQ- 9 total score $\leq 9$ points at month 2 but worsened to a PHQ-9 total score $\geq 10$ points at month 6, and "no" if PHQ-9 total score $\leq 9$ points both at month 2 and month 6 [for replacement of missing values, see Supplementary materials].

Abbreviations: DSST, Symbol Digit Substitution Test; HAM-D 17, Hamilton Depression Rating Scale - 17 items; PDQ-D, Perceived Deficits Questionnaire - Depression; PHQ-9, Patient Health Questionnaire-9. 


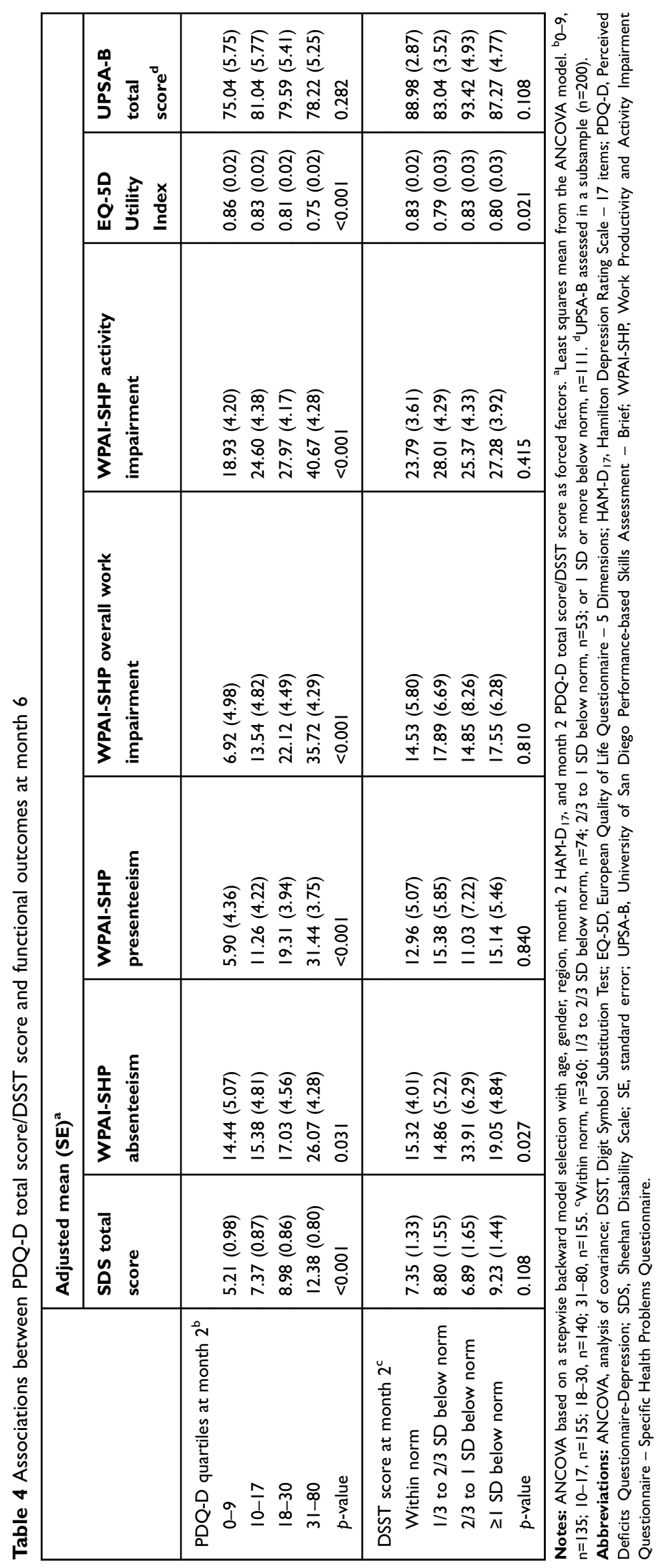




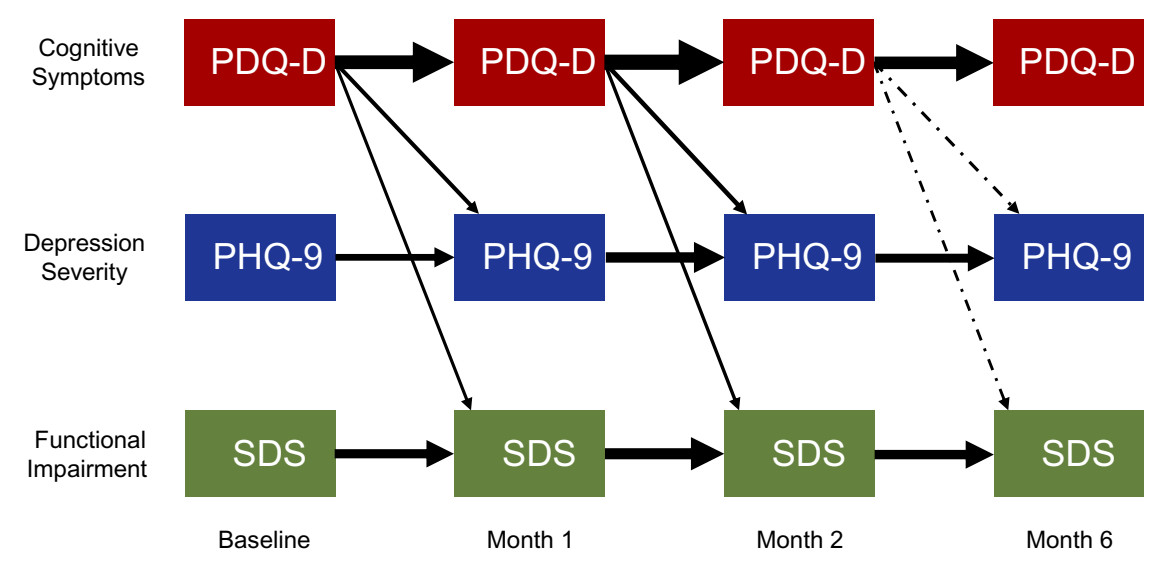

Figure 4 Temporal interdependencies among patient-reported measures of cognitive symptoms, patient-reported depressive symptoms, and functional impairment. Standardized regression coefficients based on the structural equation models (SEM) with $p$-values $<0.05$ ( $n=45 \mathrm{I}$ ). The thickness of arrows is proportional to the strength of association. A solid arrow indicates $p$-value $<0.00 \mathrm{I}$; a dot-dashed arrow indicates $p$-value $<0.05$.

Abbreviations: PDQ-D, Perceived Deficits Questionnaire - Depression; PHQ-9, Patient Health Questionnaire-9; SDS, Sheehan Disability Scale.

The mean PDQ-D total score of 34 at baseline is comparable with results from a study by Shi et al (2017) who reported a mean PDQ-D total score of 30.3 among Chinese patients with depression, as compared with a normative mean score of 9.3 among a sample of community volunteers. Shi et al (2017) further reported standardized mean differences between community volunteers and patients with depression of $0.67 \quad(p<0.01)$ for DSST [120 $\mathrm{s}$ version] and $-1.46(p<0.01)$ for PDQ-D, thus validating both measures as suited for capturing cognitive symptoms and suboptimal cognitive performance among Chinese patients with MDD. The baseline DSST performance of 50.2 is approximately 9 points higher than those observed in previous studies that included European or US patient samples with comparable levels of depression severity. ${ }^{32,33}$ This difference could reflect the general finding of slightly higher cognitive test scores among Asian versus Caucasian ethnic groups, (Weiss, 2010), but more importantly the high educational level of high school or higher for approximately three-thirds of the current sample, with almost half of patients holding a university or post-graduate degree.

Although the SEM analyses showed that improvements in cognitive symptoms (PDQ-D) predicted functioning (SDS) independently of improvements in patient-reported mood symptoms (PHQ-9), it cannot be inferred from this study that improvements in cognitive symptoms were independent of improvements mood symptoms. However, the results of the study confirm the important role of cognitive symptoms for clinical and functional outcomes reported in the literature. ${ }^{12,41,42}$

\section{Limitations}

This study was conducted in a Chinese population of patients with MDD, which largely consisted of welleducated urban residents; therefore, the results cannot necessarily be generalized to the background population of Chinese patients with MDD. With $40 \%$ of the total enrolled population being lost to follow-up at month 2, some degree of attrition bias cannot be excluded. However, the observed sociodemographic and baseline clinical characteristics between the total population, and the analysis population were very similar, which indicates that the impact on the results is likely to be negligible.

\section{Conclusions}

In this real-world study, 3 out of 4 patients with moderate-tosevere depression experienced cognitive symptoms at baseline. After 6 months of treatment, cognitive symptoms were less prevalent but still reported by nearly one-third of patients. Perceived cognitive symptoms predicted clinical outcomes, including the risk of relapse, as well as functional outcomes. Cognitive symptoms and cognitive performance were uncorrelated at baseline, and differentially associated with clinical and functional outcomes, thus showing the relevance of complementary assessment methods to fully capture all aspects of cognitive symptoms in patients with depression. The results highlight cognitive symptoms as an independent treatment goal that should be targeted directly and continuously in the treatment of MDD, in order to increase patients' chances of recovery and of restoring functioning. 


\section{Abbreviation list}

ANCOVA, analysis of covariance; CGI-S, Clinical Global Impressions - Severity of Illness; CFI, comparative fit index; DSM-5, Diagnostic and Statistical Manual of Mental Disorders, 5th Edition; DSST, Digit Symbol Substitution Test; EQ-5D, European Quality of Life Questionnaire - 5 Dimensions; HAM- $\mathrm{D}_{17}$, Hamilton Depression Rating Scale - 17 Items; ICD-10, International Classification of Diseases and Related Health Problems, 10th revision; MDD, major depressive disorder; PDQ-D, Perceived Deficits Questionnaire Depression; PHQ-9, Patient Health Questionnaire - 9 items; PROACT, the Prospective Research Observation to Assess Cognition in Treated MDD patients; RMSEA, root mean square error of approximation; SE, standard error; SEM, structural equation modeling; SDS, Sheehan Disability Scale; SRC, standardized regression coefficients; UPSA-B, University of San Diego Performancebased Skills Assessment - Brief; WPAI-SHP, Work Productivity and Activity Impairment - Specific Health Problems Questionnaire.

\section{Data availability}

The authors declare that the data supporting the findings of this study are available within the article. The authors may be contacted for further data sharing.

\section{Acknowledgments}

The authors wish to thank all patients who took part in this study, as well as the investigators and study personnel at the following sites: Beijing An Ding Hospital, Capital Medical University; No.6 Hospital of Beijing University; Shanghai Mental Health Centre, Shanghai Jiao Tong University School of Medicine; The 2nd Xiangya Hospital of Central South University; West China Hospital, Sichuan University; Nanjing Brain Hospital; Beijing Huilongguan Hospital; Shenzhen Mental Health Center; The First Affiliated Hospital of Kunming Medical College; Tianjin Anding Hospital; Beijing Tian Tan Hospital; First Affiliated Hospital of Medical College of Xi'an Jiaotong University; Beijing Chaoyang Hospital; The 3rd People's Hospital of Huzhou City; and The 3rd Hospital of Sun Yat-Sen University.

\section{Disclosure}

The analyses reported in this paper were presented at the 31st European College of Neuropsychopharmacology
Annual Meeting 2018 as poster presentations with interim findings. Posters' abstracts were published in European Neuropsychopharmacology 2019: www.sciencedirect. com/journal/european-neuropsychopharmacology/vol/29/ $\operatorname{suppl} / \mathrm{S} 1$.

H. Lundbeck A/S and Lundbeck China sponsored the study and supported this publication.

G Wang has received honoraria for being an advisor to or providing educational talks for Lundbeck, Pfizer, Sumitomo, Janssen and Janssen, and Eli Lilly company.

TM Si has been a consultant and/or advisor to Janssen Research and Development (Beijing), Pfizer, Lundbeck, and Otsuka. She received honoraria and/or grant support from Janssen Research and Development (Beijing), Lundbeck, Pfizer, and Otsuka.

CX Wang has received honoraria for being an advisor to or providing educational talks for Lundbeck, Lilly and Pfizer Company.

LN Wang has received honoraria for being an advisor to or providing educational talks for Lundbeck, Pfizer, Sumitomo, Eli Lilly Company.

Y Fang has been a consultant and/or advisor to Janssen \& Janssen, Pfizer, Lundbeck, Eli Lilly, and Otsuka. He received grant support from Eli Lilly Company.

$\mathrm{L} \mathrm{Li}$ has received honoraria for being an advisor to or providing educational talks for Lundbeck, Pfizer, Janssen Company.

A Ettrup and HLF Eriksen are full-time employees of Lundbeck. KHX Tan and L Ge were full-time employees of Lundbeck Singapore and Lundbeck China, respectively, at the time of study conduct.

The authors report no other conflicts of interest in this work.

\section{References}

1. World Health Organization. Depression and Other Common Mental Disorders: Global Health Estimates. Geneva: World Health Organization; 2017.

2. Gu L, Xie J, Long J, et al. Epidemiology of major depressive disorder in Mainland China: a systematic review. PLoS One. 2013;8(6):e65356. doi:10.1371/journal.pone.0065356

3. Lee S, Tsang A, Huang YQ, et al. The epidemiology of depression in metropolitan China. Psychol Med. 2008;39(5):735-747. doi:10.1017/ S0033291708004091

4. Lim GY, Tam WW, Lu Y, Ho CS, Zhang MW, Ho RC. Prevalence of depression in the community from 30 countries between 1994 and 2014. Sci Rep. 2018;8(1):2861. doi:10.1038/s41598-018-21243-x

5. American Psychiatric Association. Diagnostic and Statistical Manual of Mental Disorders. Fifthed. DSM-5. Washington DC: American Psychiatric Publishing; 2013.

6. Srisurapanont M, Hong JP, Tian-Mei S, et al. Clinical features of depression in Asia: results of a large prospective, cross-sectional study. Asia Pac Psychiatry. 2013;5(4):259-267. doi:10.1111/appy.12104 
7. World Health Organisation. ICD-10 Classifications of Mental and Behavioural Disorder: Clinical Descriptions and Diagnostic Guidelines. Geneva: World Health Organisation; 1992.

8. Srisurapanont M, Bautista D, Chen C-H, Wang G, Udomratn P, Eurviriyanukul K. Subjective memory and concentration deficits in medication-free, non-elderly Asians with major depressive disorder: prevalence and their correlates. J Affect Disord. 2015;171:105-110. doi:10.1016/j.jad.2014.09.007

9. Conradi HJ, Ormel J, de Jonge P. Presence of individual (residual) symptoms during depressive episodes and periods of remission: a 3-year prospective study. Psychol Med. 2011;41(6):1165-1174. doi: $10.1017 / \mathrm{S} 0033291710001911$

10. Greer TL, Kurian BT, Trivedi MH. Defining and measuring functional recovery from depression. CNS Drugs. 2010;24(4):267-284. doi:10.2165/11530230-000000000-00000

11. Vicent-Gil M, Keymer-Gausset A, Serra-Blasco M, et al. Cognitive predictors of illness course at 12 months after first-episode of depression. Eur Neuropsychopharmacol. 2018;28(4):529-537. doi:10.1016/j.euroneuro.2018.02.001

12. Papakostas GI. Cognitive symptoms in patients with major depressive disorder and their implications for clinical practice. $J$ Clin Psychiatry. 2014;75(1):8-14. doi:10.4088/JCP.13r08710

13. McIntyre RS, Lee Y, Mansur RB. Treating to target in major depressive disorder: response to remission to functional recovery. CNS Spectr. 2015;20(Suppl 1):20-30; quiz 31. DOI:10.1017/S1092852915000826

14. Woo YS, Rosenblat JD, Kakar R, Bahk WM, McIntyre RS. Cognitive deficits as a mediator of poor occupational function in remitted major depressive disorder patients. Clin Psychopharmacol Neurosci. 2016;14(1):1-16. doi:10.9758/cpn.2016.14.1.1

15. McIntyre RS, Cha DS, Soczynska JK, et al. Cognitive deficits and functional outcomes in major depressive disorder: determinants, substrates, and treatment interventions. Depress Anxiety. 2013;30 (6):515-527. doi:10.1002/da.22063

16. McIntyre RS, Soczynska JZ, Woldeyohannes HO, et al. The impact of cognitive impairment on perceived workforce performance: results from the international mood disorders collaborative project. Compr Psychiatry. 2015;56:279-282. doi:10.1016/j.comppsych.2014.08.051

17. Hu TW, He Y, Zhang M, Chen N. Economic costs of depression in China. Soc Psychiatry Psychiatr Epidemiol. 2007;42(2):110-116. doi:10.1007/s00127-006-0151-2

18. Fehnel SE, Forsyth BH, DiBenedetti DB, Danchenko N, François C, Brevig T. Patient-centered assessment of cognitive symptoms of depression. CNS Spectr. 2013;21(1):43-52. doi:10.1017/S1092852 913000643

19. Shi C, Wang G, Tian F, et al. Reliability and validity of Chinese version of perceived deficits questionnaire for depression in patients with MDD. Psychiatry Res. 2017;252:319-324. doi:10.1016/j. psychres.2017.03.021

20. Jaeger J. Digit symbol substitution test: the case for sensitivity over specificity in neuropsychological testing. J Clin Psychopharmacol. 2018;38(5):513-519. doi:10.1097/JCP.0000000000000941

21. Wechsler D. Wechsler Adult Intelligence Scale. 3rd ed. San Antonio, TX: Psychological Corporation; 1997.

22. McIntyre RS, Harrison J, Loft H, Jacobson W, Olsen CK. The effects of vortioxetine on cognitive function in patients with major depressive disorder: a meta-analysis of three randomized controlled trials. Int J Neuropsychopharmacol. 2016;19(10):pyw055. doi:10.1093/ijnp/ pyw055

23. Hamilton M. A rating scale for depression. J Neurol Neurosurg Psychiatry Res. 1960;23(1):56-62. doi:10.1136/jnnp.23.1.56

24. Guy W. Clinical Global Impressions. In: Guy W, editor. ECDEU Assessment Manual for Psychopharmacology Revised. Rockville, MD: National Institute of Mental Health; 1976:217-222.
25. Kroenke K, Spitzer RL, Williams JB. The PHQ-9: validity of a brief depression severity measure. J Gen Intern Med. 2001;16(9):606-613. 26. Mausbach BT, Harvey PD, Goldman SR, Jeste DV, Patterson TL. Development of a brief scale of everyday functioning in persons with serious mental illness. Schizophr Bull. 2007;33(6):1364-1372. doi:10.1093/schbul/sbm014

27. Reilly MC, Zbrozek AS, Dukes EM. The validity and reproducibility of a work productivity and activity impairment instrument. PharmacoEconomics. 1993;4(5):353-365. doi:10.2165/00019053199304050-00006

28. The EuroQoL Group. EuroQol - a new facility for the measurement of health-related quality of life. Health Policy. 1990;16(3):199-208.

29. Wechsler D. Wechsler Adult Intelligence Scale-Revised. San Antonio, TX: Psychological Corporation; 1981.

30. Bollen KA. Structural Equations with Latent Variables. Hoboken, NJ: Wiley; 1989.

31. Rosseel Y. lavaan: an R Package for structural equation modeling. J Stat Softw. 2012;48(2):36.

32. Mahableshwarkar AR, Zajecka J, Jacobson W, Chen Y, Keefe RS. A randomized, placebo-controlled, active-reference, double-blind, flexible-dose study of the efficacy of vortioxetine on cognitive function in major depressive disorder. Neuropsychopharmacology. 2015;40(8):2025-2037. doi:10.1038/npp.2015.52

33. McIntyre RS, Lophaven S, Olsen CK. A randomized, double-blind, placebo-controlled study of vortioxetine on cognitive function in depressed adults. Int $J$ Neuropsychopharmacol. 2014;17 (10):1557-1567. doi:10.1017/S1461145714000546

34. Rosenblat JD, Kakar R, McIntyre RS. The cognitive effects of antidepressants in major depressive disorder: a systematic review and meta-analysis of randomized clinical trials. Int J Neuropsychopharmacol. 2016;19:2. doi:10.1093/ijnp/pyv082

35. Baune BT, Brignone M, Larsen KG. A network meta-analysis comparing effects of various antidepressant classes on the Digit symbol substitution test (DSST) as a measure of cognitive dysfunction in patients with major depressive disorder. Int J Neuropsychopharmacol. 2017;21(2):97-107.

36. Marino SE, Meador KJ, Loring DW, et al. Subjective perception of cognition is related to mood and not performance. Epilepsy Behav. 2009;14(3):459-464. doi:10.1016/j.yebeh.2008.12.007

37. Srisurapanont M, Suttajit S, Eurviriyanukul K, Varnado P. Discrepancy between objective and subjective cognition in adults with major depressive disorder. Sci Rep. 2017;7(1):3901. doi:10.1038/s41598-017-04353-w

38. Crumley JJ, Stetler CA, Horhota M. Examining the relationship between subjective and objective memory performance in older adults: a meta-analysis. Psychol Aging. 2014;29(2):250-263. doi: $10.1037 / \mathrm{a} 0035908$

39. van der Werf-Eldering MJ, Burger H, Jabben N, Holthausen EAE, Aleman A, Nolen WA. Is the lack of association between cognitive complaints and objective cognitive functioning in patients with bipolar disorder moderated by depressive symptoms? J Affect Disord. 2011;130(1):306-311. doi:10.1016/j.jad.2010.10.005

40. Hammer-Helmich L, Haro JM, Jonsson B, et al. Functional impairment in patients with major depressive disorder: the 2-year PERFORM study. Neuropsychiatr Dis Treat. 2018;14:239-249. doi:10.2147/NDT.S146098

41. Lam RW, Kennedy SH, McLntyre RS, Khullar A. Cognitive dysfunction in major depressive disorder: effects on psychosocial functioning and implications for treatment. Can J Psychiatry. 2014;59 (12):649-654. doi:10.1177/070674371405901206

42. Larsen KG, Haro JM, Saragoussi D, Hammer-Helmich L. Depression severity and cognitive symptoms are independent predictors of functioning in patients with depression. Eur Neuropsychopharmacol. 2017;27:S806. doi:10.1016/S0924-977X(17)31461-X 


\section{Supplementary materials}

Selection procedure for logistic regression and ANCOVA models for clinical and functional outcomes

A series of univariate analyses were conducted for a list of candidate explanatory variables (see below), which were identified based on a literature review and clinical experience. Next, for each outcome variable, a backward stepwise procedure, ie, sequential removal of the least significant variable from the model and re-estimating the model until all independent variables were statistically significant at $p<0.05$, was applied on statistically significant variables ( $p$-value $<0.20$ ) in the univariate models.

Univariate analyses with response at month 1 and remission at month 2 comprised the factors listed in i) + ii) + iii). Univariate analyses with remission and relapse at month 6 comprised the factors listed in i) + ii) + iv) + v). Univariate analyses with functional outcomes at month 6 comprised the factors listed in i) + iv).

i) Age at baseline (18-25, 26-34, 35-54, 55-65 years); sex at baseline (male, female); region at baseline (North, South, East, West); educational level at baseline (no degree or diploma/elementary school/middle school, high school/junior college, university or above); employment status at baseline (paid employment or self-employed, unemployed/student/non-working spouse/retired/disability pension/others); at least one other concomitant mental condition at baseline (yes, no); chronic pain or fibromyalgia at baseline (yes, no).

ii) Previous depressive episode at baseline (yes, no); at least one chronic medical condition at baseline (yes, no).

iii) Body mass index at baseline $\left(<30 \mathrm{~kg} / \mathrm{m}^{2}, \geq 30 \mathrm{~kg} / \mathrm{m}^{2}\right)$; tobacco use at baseline (yes, no); marital status at baseline (single/divorced/separated/widowed, married or living with partner); living area at baseline (rural, urban); switch of antidepressant at baseline (yes, no); duration of current depressive episode at baseline ( $<8$ weeks, $\geq 8$ weeks); anxiety disorder at baseline (yes, no); suicide attempt before baseline (yes, no); hospitalization for depression over the past 12 weeks before baseline (yes, no); sick-leave within 12 months before baseline (yes, no); current psychotherapy at baseline (yes, no); Clinical Global Impression Scale - Severity of Illness (CGI-S) score at baseline (1-4, 5-7); Hamilton Depression Rating Scale - 17 items $\left(\mathrm{HAM}-\mathrm{D}_{17}\right)$ total score at baseline (17-23, 24-52); Patient Health Questionnaire - 9 items (PHQ-9) total score at baseline (0-4, 5-9, 10-14, 15-19, 20-27); Digit Symbol Substitution Test (DSST) score at baseline (within norm, $1 / 3$ to $2 / 3 \mathrm{SD}$ below norm, $2 / 3$ to $1 \mathrm{SD}$ below norm, $1 \mathrm{SD}$ or more below norm); Perceived Deficits Questionnaire - Depression (PDQ-D) total score at baseline (0-Q1, Q1-median, median-Q3, Q3-80); Work Productivity and Activity Impairment - Specific Health Problems (WPAI-SHP) Activity impairment at baseline (continuous); European Quality of Life Questionnaire - 5 Dimensions (EQ-5D) utility score at baseline (continuous).

iv) Suicide attempt before baseline or between baseline and month 2 (yes, no); hospitalization for depression over the past 12 weeks before baseline or between baseline and month 2 (yes, no); sick-leave within 12 months before baseline or between baseline and month 2 (yes, no); treatment line at month 2 (1, $\geq 2$ ); discontinuation of antidepressant treatment between baseline and month 2 (yes, no); CGI-S score at month $2(1-3,4,5-7)$; HAM-D 17 total score at month 2 (0-7, 8-16 17-23, 24-52); PHQ-9 total score at month $2(0-4,5-9,10-14,15-19$, 20-27); DSST score at month 2 (within norm, 1/3 to $2 / 3 \mathrm{SD}$ below norm, $2 / 3$ to $1 \mathrm{SD}$ below norm, 1 $\mathrm{SD}$ or more below norm); PDQ-D total score at month 2 (0-Q1, Q1-median, median-Q3, Q3-80).

v) Previous or current psychotherapy at baseline (yes, no); WPAI-SHP Activity impairment at month 2 (continuous); EQ-5D utility score at month 2 (continuous).

\section{Replacement of missing values for coding of relapse}

PHQ-9 total score $\leq 9$ points at month 2: replaced with month 2 HAM-D $_{17}$ total score $\leq 7$ if month 2 PHQ-9 total score was missing, or with month 2 CGI-S score $\leq 2$ if month 2 HAM-D 17 was missing; PHQ-9 total score $\geq 10$ points at month 6 : replaced with month $6 \mathrm{HAM}-\mathrm{D}_{17}$ total score $\geq 17$ if month 6 PHQ-9 total score was missing, or with month 6 CGI-S score $\geq 4$ if month 6 HAM-D 17 was missing; PHQ-9 total score $\leq 9$ points at month 2 and month 6: replaced with $H A M-D_{17}$ total score $<17$ if PDQ-9 for these timepoints was missing, or with CGI-S score $<4$ if $\mathrm{HAM}-\mathrm{D}_{17}$ was missing. 


\section{Publish your work in this journal}

Neuropsychiatric Disease and Treatment is an international, peerreviewed journal of clinical therapeutics and pharmacology focusing on concise rapid reporting of clinical or pre-clinical studies on a range of neuropsychiatric and neurological disorders. This journal is indexed on PubMed Central, the 'PsycINFO' database and CAS, and is the official journal of The International Neuropsychiatric Association (INA). The manuscript management system is completely online and includes a very quick and fair peer-review system, which is all easy to use. Visit http://www.dovepress.com/testimonials.php to read real quotes from published authors. 